\title{
Effect of Plant Densities and Cycocel on Fruit and Yield Attributes of Okra (Abelmoschus Esculentus. (L.) Moench) Cv. Parbhani Kranti
}

\author{
M. Jyothi* and T. B. Tambe \\ Department of Horticulture, Vasantrao Naik Marathwada Krishi Vidyapeeth, \\ Parbhani, (M.S.)-431 402, India \\ *Corresponding author
}

\section{A B S T R A C T}

\section{Keywords}

Plant density, Cycocel, Spacing, Okra, Yield

\section{Article Info}

Accepted:

07 September 2019

Available Online:

10 October 2019
An experiment was conducted at College of Horticulture, Vasantrao Naik Marathwada Krishi Vidyapeeth, Parbhani, during 2018 to know the effect of different plant densities $(60 \times 30 \mathrm{~cm}, 60 \times 25 \mathrm{~cm}, 40 \times 30 \mathrm{~cm}$ and $40 \times 25 \mathrm{~cm})$ and foliar spray of cycocel $(0,400$, 600 and $800 \mathrm{ppm}$ ) on various fruit and yield attributes of okra. Experiment was laid in Factorial Randomized Block Design with three replications during summer season under shadenet house. The observations on fruit and yield attributes were recorded during investigation. Results revealed, that the significant effect of spacing levels on number of fruits per plant, fruit length, fruit yield per plot and fruit yield per hectare. Whereas, cycocel levels were found significant for the traits like days to first fruit set, days to first harvest, number of fruits per plant, average fruit weight, fruit length, fruit yield per plot and fruit yield per hectare. In case of interaction effects the characters like number of fruits per plant, average fruit weight, fruit length, fruit yield per plot and fruit yield per hectare was found significant. Remaining traits were not showed any significant performance under the experiment. The important characters like number of fruits per plant (9.38), average fruit weight $(17.93 \mathrm{~g})$, fruit length $(8.26 \mathrm{~cm})$, fruit yield per plot $(10.28 \mathrm{~kg})$ and fruit yield per hectare $(16.13 \mathrm{Mt})$, were performed significantly maximum at $40 \times 25 \mathrm{~cm}$ spacing with the foliar application of cycocel at $600 \mathrm{ppm}$. However, these characters were at par with the spacing $40 \times 30 \mathrm{~cm}$ with the foliar application of cycocel at $800 \mathrm{ppm}$. Plant density either at $40 \times 25 \mathrm{~cm}$ with cycocel at $600 \mathrm{ppm}$ or $40 \times 30 \mathrm{~cm}$ with cycocel at 800 ppm at 30 and 45 DAS was found better for yield in okra.

\section{Introduction}

Okra (Abelmoschus esculentus (L.) Moench) is an annual vegetable crop belongs to the family Malvaceae. It's adaptability to a wide range of growing condition made it popular among vegetable growers. Okra crop requires long warm growing season and it is susceptible to frost. The optimum day temperature for its better growth is between 25 to $40^{\circ} \mathrm{C}$ and that of night is over $22^{\circ} \mathrm{C}$. It thrives in all kinds of soils but to well drained medium black and light clayey soils, rich in organic matter and favourable soil $\mathrm{pH}$ range 
from 6.0 to 6.8 is more suitable for okra cultivation.

Increasing plant population increases yield per unit area of okra till a certain limit, beyond which resources for plant become limited and the yield decreases (Weimer, 1990). Planting with proper spacing increases yield quality and size of fruit. It has been observed that suitable planting spacing can lead to optimum okra fruit yield while improper planting spacing could result in relatively low yield and poor quality fruits (Maurya et al., 2013). Hossain et al., (2001) recommended that intrarow spacing for optimal okra fruit yield ranged from $20 \mathrm{~cm}$ to $40 \mathrm{~cm}$. Therefore, optimum plant population is required for higher yields of okra.

Suitable planting spacing and cycocel can lead to optimum okra fruit yield while improper planting spacing could result in relatively low yield and poor quality fruits (Maurya et al., 2013). The present investigation was undertaken by aiming to produce better more yield of okra with specific standards viz. tenderness, 3 to $4 \mathrm{~cm}$ in length, uniform in size, dark green in colour, disease and pest free with better yield potential per unit area. Cycocel is growth retardant that inhibits both cell elongation and cell division It plays an important role of anti-gibberellin growth retardant. Appropriate plant densities coupled with foliar application of cycocel resulted in increasing fruit yield and quality in okra. Therefore, the present investigation was undertaken to study the effect of plant densities and cycocel on fruit and yield attributes of okra.

\section{Materials and Methods}

The investigation was undertaken at Instructional cum Research Farm of College of Horticulture, Vasantrao Naik Marathwada Krishi Vidyapeeth, Parbhani to know the effect of plant densities and cycocel on fruit and yield attributes of okra. The experiment was laid out in Factorial Randomized Block Design with three replications during summer season of 2018 under shade net house. Sowing was undertaken at four different spacing levels i.e $60 \times 30 \mathrm{~cm}, 60 \times 25 \mathrm{~cm}, 40 \times 30 \mathrm{~cm}$ and 40 $\mathrm{x} 25 \mathrm{~cm}$. Whereas, cycocel was applied as foliar spray at 30 and 45 days after sowing (DAS) at four different concentration levels i.e. 0 ppm (distilled water), 400, 600 and 800 ppm. All, recommended practices like nutrient and plant protection measures were given during the experiment. Different commercial characters of okra were recorded during growth of the crop viz. days to first fruit set, days to first fruit harvest, number of fruits per plant, average fruit weight, fruit length, fruit diameter, fruit yield per plant, fruit yield per plot and fruit yield per hectare. Collected information was subjected to statistical analysis to test the significance among the treatments on various characters of okra under study was done according to the procedure given by Panse and Sukhatme (1985).

\section{Results and Discussion}

\section{Effect of plant density}

The results of the experiment indicated that the significant effect of different plant densities were shown for the characters like number of fruits per plant, fruit length, fruit yield per plot and fruit yield per hectare. Whereas, other characters like days to first fruit set, days to first fruit harvest, average fruit weight, fruit diameter and fruit yield per plant were found non significant (Table 1). Number of fruits per plant were recorded significantly more at spacing of $40 \times 25 \mathrm{~cm}$ (8.50) followed by 40 x $30 \mathrm{~cm} \mathrm{(8.40),}$ whereas, fruit length was found significantly maximum in $40 \times 30 \mathrm{~cm}$ (7.63) followed by 60 x $30 \mathrm{~cm}$ and $60 \times 25 \mathrm{~cm}$ i.e. $7.61 \mathrm{~cm}$ and 7.54 $\mathrm{cm}$, respectively. Characters like fruit yield 
per plot $(7.86 \mathrm{~kg})$ and fruit yield per hectare $(13.36 \mathrm{Mt})$ was recorded at spacing of $40 \times 25$ $\mathrm{cm}$ followed by $40 \times 30 \mathrm{~cm}(7.31 \mathrm{~kg}$ and 12.29 $\mathrm{Mt}$, respectively). Similar results were also reported by Maurya et al., (2013) and Ekwu et al., (2012).

\section{Effect of cycocel}

Cycocel showed significant effect on different characters of okra like days to first fruit set, days to first fruit harvest, number of fruits per plant, average fruit weight, fruit length, fruit diameter, fruit yield per plot and fruit yield per hectare, whereas, fruit yield per plant was not shown more difference among the treatments. Number of fruits per plant was found significantly maximum (8.50) at floiar application of cycocel at $600 \mathrm{ppm}$ followed by 800 ppm (7.90). Average fruit weight, fruit length, fruit diameter, fruit yield per plot and fruit yield per hectare were recorded maximum at foliar application of cycocelat $600 \mathrm{ppm}$ i.e. $18.75 \mathrm{~g}, 7.61 \mathrm{~cm}, 18.75 \mathrm{~cm}, 7.65$ $\mathrm{kg}$ and $12.69 \mathrm{Mt}$, respectively. These findings are in agreement with those of Singh (2013), Rajput et al., (2011), Munikrishnappa and Shantappa (2009), Pateliya (2008) and Parmar et al., (2008).

\section{Effect of interaction}

Interaction effects revealed that the traits like number of fruits per plant, average fruit weight, fruit length, fruit yield per plot and fruit yield per hectare was found significant, while other characters like days to first fruit set, days to first fruit harvest, fruit diameter and fruit yield per plant were not shown any more differences among the treatments (Table 2).

Number of fruits per plant was exhibited maximum and significant number (9.38) at $\mathrm{S}_{4} \mathrm{C}_{3}$ i.e. spacing at $40 \times 25 \mathrm{~cm}$ with the foliar application of cycocel at $600 \mathrm{ppm}$. Whereas,
$\mathrm{S}_{4} \mathrm{C}_{4}(40 \times 25 \mathrm{~cm}$, cycocel at $800 \mathrm{ppm}), \mathrm{S}_{3} \mathrm{C}_{3}$ $(40 \times 30 \mathrm{~cm}$, cycocel at $600 \mathrm{ppm})$ and $\mathrm{S}_{3} \mathrm{C}_{4}$ $(40 \times 30 \mathrm{~cm}$, cycocel at $800 \mathrm{ppm})$ were also found at par with $\mathrm{S}_{4} \mathrm{C}_{3}(40 \times 25 \mathrm{~cm}$, cycocel at 600 ppm) i.e. $9.16,9.14$ and 9.05 fruits per plant per ha, respectively.

The characters like average fruit weight, fruit length, fruit yield per plot and fruit yield per hectare were also recorded significantly maximum in $\mathrm{S}_{4} \mathrm{C}_{3}(40 \times 25 \mathrm{~cm}$,cycocel at 600 ppm) viz. $17.93 \mathrm{~g}, 8.26 \mathrm{~cm}, 10.28 \mathrm{~kg}$ and 16.13 Mt, respectively. Audus (1960) and Kakade et al., (2010) were also reported similar findings.

Among the treatments, significantly maximum yield potential was reported from $\mathrm{S}_{4} \mathrm{C}_{3}(40 \mathrm{x}$ $25 \mathrm{~cm}$ with the foliar application of cycocel at $600 \mathrm{ppm}$ ) treatment recorded $16.13 \mathrm{Mt}$ per hectare and whereas, plant density at $\mathrm{S}_{4} \mathrm{C}_{4}(40$ $\mathrm{x} 25 \mathrm{~cm}$ with the foliar application of cycocel at $800 \mathrm{ppm})$ and $\mathrm{S}_{3} \mathrm{C}_{3}(40 \times 30 \mathrm{~cm}$ with foliar application of cycocel at $600 \mathrm{ppm}$ ) were also found at par with $\mathrm{S}_{4} \mathrm{C}_{3}(40 \times 25 \mathrm{~cm}$ with the foliar application of cycocel at $600 \mathrm{ppm}$ ) with yield level of $15.97 \mathrm{Mt}$ and 15.53 Mt per hectare, respectively followed by $\mathrm{S}_{3} \mathrm{C}_{4}(40 \mathrm{x}$ $30 \mathrm{~cm}$ with the foliar application of cycocel at $800 \mathrm{ppm}$ ) i.e. $14.11 \mathrm{Mt}$ per hectare.

Whereas, $\mathrm{S}_{1} \mathrm{C}_{4}(60 \mathrm{x} 30$ with the foliar application of cycocel at $800 \mathrm{ppm})$ and $\mathrm{S}_{1} \mathrm{C}_{1}$ $(60 \times 30$ without the foliar application of cycocel recorded significantly, the lowest i.e. 10.11 Mt and 10.24 Mt per hectare yield level out of all the treatments under study. Similar results were also reported by Maurya et al., (2013) and Ekwu et al., (2012).

It is concluded that the spacing at $40 \times 25 \mathrm{~cm}$ with the foliar applications of cycocel at 600 ppm at 30 and 45 DAS or spacing at $40 \times 30$ $\mathrm{cm}$ with the foliar applications of cycocel at $800 \mathrm{ppm}$ at 30 and 45 DAS was found better for fruit yield in okra. 
Table .1 Effect of plant densities and cycocel on fruit and yield attributes in okra

\begin{tabular}{|c|c|c|c|c|c|c|c|c|c|c|}
\hline \multicolumn{2}{|c|}{$\begin{array}{c}\text { Traits/ } \\
\text { Treatment details }\end{array}$} & \multirow{2}{*}{$\begin{array}{c}\begin{array}{c}\text { Days to } \\
\text { first fruit } \\
\text { set }\end{array} \\
43.85\end{array}$} & \multirow{2}{*}{$\begin{array}{c}\begin{array}{c}\text { Days to } \\
\text { first fruit } \\
\text { harvest }\end{array} \\
51.17\end{array}$} & \multirow{2}{*}{$\begin{array}{c}\begin{array}{c}\text { No. of } \\
\text { fruits per } \\
\text { plant }\end{array} \\
6.41^{\mathrm{c}} \\
\end{array}$} & \multirow{2}{*}{$\begin{array}{c}\begin{array}{c}\text { Average } \\
\text { fruit weight } \\
(\mathbf{g})\end{array} \\
16.80\end{array}$} & \multirow{2}{*}{$\begin{array}{c}\begin{array}{c}\text { Fruit length } \\
\text { (cm) }\end{array} \\
7.61^{\mathrm{a}}\end{array}$} & \multirow{2}{*}{$\begin{array}{c}\begin{array}{c}\text { Fruit } \\
\text { diameter } \\
(\mathbf{m m})\end{array} \\
12.59\end{array}$} & \multirow{2}{*}{$\begin{array}{c}\begin{array}{c}\text { Fruit yield } \\
\text { per plant } \\
\text { (g) }\end{array} \\
115.11 \\
\end{array}$} & \multirow{2}{*}{$\begin{array}{c}\begin{array}{c}\text { Fruit yield per } \\
\text { plot (kg) }\end{array} \\
6.14^{\mathrm{d}}\end{array}$} & \multirow{2}{*}{$\begin{array}{c}\begin{array}{c}\text { Fruit yield per } \\
\text { hectare } \\
\text { (MT/ha.) }\end{array} \\
10.15^{\mathrm{d}}\end{array}$} \\
\hline$S_{1}$ & $60 \times 30 \mathrm{~cm}$ & & & & & & & & & \\
\hline $\mathbf{S}_{2}$ & $60 \times 25 \mathrm{~cm}$ & 43.28 & 51.11 & $7.60^{\mathrm{b}}$ & 16.96 & $7.54^{\mathrm{a}}$ & 12.74 & 117.20 & $6.53^{c}$ & $10.86^{\mathrm{c}}$ \\
\hline$S_{3}$ & $40 \times 30 \mathrm{~cm}$ & 43.12 & 50.98 & $8.40^{\mathrm{a}}$ & 17.00 & $7.63^{\mathrm{a}}$ & 12.75 & 118.31 & $7.31^{\mathrm{b}}$ & $12.29^{\mathrm{b}}$ \\
\hline$S_{4}$ & $40 \times 25 \mathrm{~cm}$ & 42.48 & 50.53 & $8.50^{\mathrm{a}}$ & 17.27 & $7.28^{\mathrm{b}}$ & 12.88 & 122.93 & $7.86^{\mathrm{a}}$ & $13.36^{\mathrm{a}}$ \\
\hline \multicolumn{2}{|c|}{ C.D@ 5\% } & NS & NS & $0.720 *$ & NS & $0.177 *$ & NS & NS & $0.375 *$ & $0.591 *$ \\
\hline $\mathbf{C}_{1}$ & $0 \mathrm{ppm}$ & $43.78^{\mathrm{a}}$ & $51.70^{\mathrm{a}}$ & $6.30^{\mathrm{b}}$ & $16.63^{d}$ & $7.28^{\mathrm{b}}$ & $16.63^{\mathrm{d}}$ & 113.36 & $6.02^{\mathrm{c}}$ & $10.11^{\mathrm{d}}$ \\
\hline $\mathrm{C}_{2}$ & $400 \mathrm{ppm}$ & $43.10^{\mathrm{a}}$ & $51.00^{\mathrm{ab}}$ & $6.80^{\mathrm{b}}$ & $17.05^{\mathrm{c}}$ & $7.57^{\mathrm{a}}$ & $17.05^{\mathrm{c}}$ & 119.15 & $6.24^{c}$ & $10.73^{\mathrm{c}}$ \\
\hline $\mathrm{C}_{3}$ & $600 \mathrm{ppm}$ & $43.05^{\mathrm{ab}}$ & $50.80^{\mathrm{b}}$ & $8.50^{\mathrm{a}}$ & $18.75^{\mathrm{a}}$ & $7.61^{\mathrm{a}}$ & $18.75^{\mathrm{a}}$ & 121.3 & $7.65^{\mathrm{a}}$ & $12.69^{\mathrm{a}}$ \\
\hline $\mathrm{C}_{4}$ & $800 \mathrm{ppm}$ & $42.80^{b}$ & $50.20^{\mathrm{b}}$ & $7.90^{\mathrm{a}}$ & $17.69^{b}$ & $7.58^{\mathrm{a}}$ & $17.69^{b}$ & 120.12 & $7.18^{b}$ & $11.68^{b}$ \\
\hline \multicolumn{2}{|c|}{ C.D@ 5\% } & $0.885^{*}$ & $0.915^{*}$ & $1.489 *$ & $0.415 *$ & $0.177 *$ & $0.359 *$ & NS & $0.375^{*}$ & 0.591* \\
\hline
\end{tabular}

Within a treatment group, means in a column followed by the same letter(s) are not significantly different using CD at $5 \%$ level of probability.

NS = Not Significant, $*=$ Significant. $S=$ Spacing levels, $\quad C=$ Cycocel levels

Table.2 Interaction effect of plant densities and cycocel on fruit and yield attributes of okra

\begin{tabular}{|c|c|c|c|c|c|c|c|c|c|c|}
\hline \multicolumn{2}{|c|}{$\begin{array}{c}\text { Traits/ } \\
\text { Treatment details }\end{array}$} & \multirow{2}{*}{$\begin{array}{c}\begin{array}{c}\text { Days to } \\
\text { first fruit } \\
\text { set }\end{array} \\
44.73\end{array}$} & \multirow{2}{*}{\begin{tabular}{|c|}
$\begin{array}{r}\text { Days to first } \\
\text { fruit harvest }\end{array}$ \\
52.80 \\
\end{tabular}} & \multirow{2}{*}{$\begin{array}{c}\begin{array}{c}\text { No. of fruits } \\
\text { per plant }\end{array} \\
7.74^{\mathrm{e}}\end{array}$} & \multirow{2}{*}{$\begin{array}{c}\begin{array}{c}\text { Average } \\
\text { fruit weight } \\
\text { (g) }\end{array} \\
15.91^{\mathrm{e}} \\
\end{array}$} & \multirow{2}{*}{$\begin{array}{c}\begin{array}{c}\text { Fruit length } \\
(\mathbf{c m})\end{array} \\
6.86^{\mathrm{h}}\end{array}$} & \multirow{2}{*}{$\begin{array}{c}\begin{array}{c}\text { Fruit } \\
\text { diameter } \\
(\mathbf{m m})\end{array} \\
12.01\end{array}$} & \multirow{2}{*}{$\begin{array}{c}\begin{array}{c}\text { Fruit yield } \\
\text { per plant }(\mathrm{g})\end{array} \\
104.18\end{array}$} & \multirow{2}{*}{$\begin{array}{c}\begin{array}{c}\text { Fruit yield per } \\
\text { plot (kg) }\end{array} \\
6.61^{\mathrm{e}} \\
\end{array}$} & \multirow{2}{*}{$\begin{array}{c}\begin{array}{c}\text { Fruit yield per } \\
\text { hectare (MT/ha.) }\end{array} \\
10.24^{\mathrm{f}} \\
\end{array}$} \\
\hline $\mathbf{T}_{1}$ & $\mathrm{~S}_{1} \mathrm{C}_{1}$ & & & & & & & & & \\
\hline $\mathbf{T}_{2}$ & $\mathrm{~S}_{2} \mathrm{C}_{1}$ & 44.47 & 51.85 & $8.07^{\mathrm{de}}$ & $16.06^{\mathrm{e}}$ & $7.03^{\mathrm{g}}$ & 12.28 & 106.09 & $6.91^{\mathrm{de}}$ & $10.4^{\mathrm{f}}$ \\
\hline $\mathbf{T}_{3}$ & $\mathrm{~S}_{3} \mathrm{C}_{1}$ & 43.87 & 51.80 & $8.16^{\mathrm{d}}$ & $16.61^{\text {de }}$ & $7.22^{f}$ & 12.45 & 111.07 & $6.96^{\mathrm{de}}$ & $10.44^{f}$ \\
\hline $\mathbf{T}_{4}$ & $\mathrm{~S}_{4} \mathrm{C}_{1}$ & 43.53 & 51.61 & $8.19^{\mathrm{d}}$ & $16.73^{\text {cde }}$ & $7.25^{f}$ & 12.44 & 115.29 & $7.03^{\mathrm{de}}$ & $10.52^{f}$ \\
\hline $\mathbf{T}_{5}$ & $\mathrm{~S}_{1} \mathrm{C}_{2}$ & 43.51 & 51.60 & $8.18^{\mathrm{d}}$ & $16.74^{\text {cde }}$ & $7.27^{\mathrm{ef}}$ & 12.65 & 116.89 & $7.11^{\mathrm{de}}$ & $10.69^{\mathrm{ef}}$ \\
\hline$T_{6}$ & $\mathrm{~S}_{2} \mathrm{C}_{2}$ & 43.33 & 51.20 & $8.17^{\mathrm{d}}$ & $16.94^{\text {bcd }}$ & $7.29^{\mathrm{ef}}$ & 12.67 & 117.31 & $7.11^{\mathrm{de}}$ & $10.76^{\mathrm{ef}}$ \\
\hline $\mathbf{T}_{7}$ & $\mathrm{~S}_{3} \mathrm{C}_{2}$ & 43.27 & 51.01 & $8.27^{\mathrm{d}}$ & $16.99^{\text {bcd }}$ & $7.29^{\text {ef }}$ & 12.71 & 117.48 & $7.23^{\mathrm{de}}$ & $10.85^{\mathrm{ef}}$ \\
\hline $\mathbf{T}_{8}$ & $\mathrm{~S}_{4} \mathrm{C}_{2}$ & 43.20 & 51.00 & $8.31^{\mathrm{d}}$ & $16.99^{\text {bcd }}$ & $7.31^{\text {ef }}$ & 12.75 & 118.94 & $7.36^{\mathrm{de}}$ & $11.83^{\mathrm{de}}$ \\
\hline$T_{9}$ & $\mathrm{~S}_{1} \mathrm{C}_{3}$ & 43.07 & 50.81 & $8.34^{\mathrm{d}}$ & $17.05^{\text {bcd }}$ & $7.38^{\mathrm{e}}$ & 12.82 & 119.71 & $7.40^{\mathrm{d}}$ & $12.07^{\mathrm{d}}$ \\
\hline$T_{10}$ & $\mathrm{~S}_{2} \mathrm{C}_{3}$ & 42.93 & 50.80 & $8.78^{c}$ & $17.08^{\text {bcd }}$ & $7.70^{\mathrm{d}}$ & 12.86 & 121.01 & $7.45^{\mathrm{d}}$ & $12.25^{\mathrm{d}}$ \\
\hline$T_{11}$ & $\mathrm{~S}_{3} \mathrm{C}_{3}$ & 42.53 & 50.20 & $9.14^{\mathrm{ab}}$ & $17.50^{\mathrm{abc}}$ & $7.95^{\mathrm{c}}$ & 12.91 & 123.69 & $9.92^{\mathrm{ab}}$ & $15.53^{\mathrm{ab}}$ \\
\hline$T_{12}$ & $\mathrm{~S}_{4} \mathrm{C}_{3}$ & 42.07 & 48.93 & $9.38^{\mathrm{a}}$ & $17.93^{\mathrm{a}}$ & $8.26^{\mathrm{a}}$ & 12.97 & 132.13 & $10.28^{\mathrm{a}}$ & $16.13^{\mathrm{a}}$ \\
\hline $\mathbf{T}_{13}$ & $\mathrm{~S}_{1} \mathrm{C}_{4}$ & 42.87 & 50.61 & $8.85^{\mathrm{b}}$ & $17.12^{\text {abcd }}$ & $7.71^{\mathrm{d}}$ & 13.00 & 121.27 & $7.45^{\mathrm{d}}$ & $10.11^{\mathrm{f}}$ \\
\hline$T_{14}$ & $\mathrm{~S}_{2} \mathrm{C}_{4}$ & 42.73 & 50.60 & $8.90^{\mathrm{b}}$ & $17.42^{\text {abcd }}$ & $7.72^{\mathrm{d}}$ & 13.02 & 121.34 & $8.84^{\mathrm{c}}$ & $13.74^{\mathrm{c}}$ \\
\hline$T_{15}$ & $\mathrm{~S}_{3} \mathrm{C}_{4}$ & 42.60 & 50.50 & $9.05^{\mathrm{abc}}$ & $17.49^{\mathrm{abc}}$ & $7.86^{\mathrm{c}}$ & 13.04 & 123.37 & $9.22^{\mathrm{bc}}$ & $14.11^{\mathrm{bc}}$ \\
\hline$T_{16}$ & $\mathrm{~S}_{4} \mathrm{C}_{4}$ & 42.20 & 49.87 & $9.16^{\mathrm{ab}}$ & $17.59^{\mathrm{ab}}$ & $8.13^{b}$ & 13.24 & 124.44 & $10.18^{\mathrm{a}}$ & $15.97^{\mathrm{a}}$ \\
\hline \multicolumn{2}{|c|}{ C.D@ 5\% } & NS & NS & $0.355^{*}$ & 0.831* & 0.122* & NS & NS & 0.751* & 1.182* \\
\hline
\end{tabular}

Within a treatment group, means in a column followed by the same letter(s) are not significantly different using CD at $5 \%$ level of probability. NS $=$ Not Significant, $*=$ Significant $\quad \mathrm{S}=$ Spacing levels, C= Cycocel levels 
References

Audus, L. J, (1960). In encydopedia of plant physiology (Ed. L. Ruhaland). Spmger Berlin, 3(2); 360-387.

Ekwu,L.G. and Nwokwu, G.N. (2012) Effect of plant spacing and planting date on the growth and yield of okra (Abelmoschus esculentus (L.) Moench). In Abakaliki. Int J. Agric. Rural Dev.

Kakade, D, K., H. B Patel, S. Rukum, G. V. Tomar, G. Kulkami, N. A. Memane, S. J. Deshmukh, Sharma and C. D. Patel (2010). Effect of plant growth regulators on sex expression and yield of sponge gourd. The Asian J. of Hort., 4(2): 408-410.

Maurya R. P., Jamar A. Bailey, Jeff St. A. Chandler. (2013) Impact of plant spacings and picking interval on the growth, fruit quality and yield of okra (Abelmoschus esculentus (L.) Moench). Ameri. J.of Agri and Forestry, 1(4): 48- 54.

Munikrishnappa, P. M. and Shantappa (2009). Influence of seed treatment and foliar application of growth regulators on growth and yield of bhendi (Abelmoschus esculentus) $\mathrm{Cv}$. Arka Anamika. J. Ecol. biology, 25(4): 323-328.

Panse, V.G. and Sukhatme, P.U. (1985). Statistical Methods for Agricultural Workers, Indian Council of Agriculture Research, New Delhi. pp:
199-216.

Parmar, B. R., C. K. Pateliy and Y. N. Tandel (2008). Effect of different growth retardants on flowering, yield and economics of okra (Abelmoschus esulentus L.) Cv. Go-2 under South Gujarat conditions. The Asian J. of Hort.,310 (2): 317-318.

Pateliya, C.K., Parmer, B.R. and Tandel, Y.N.(2008). Effect of different growth retardants on flowering, yield and economic of okra $\mathrm{Cv}$. Co-2 under South Gujarat conditions. The Asian J. of Hort.3(2): 317-318.

Rajput, B. S., Singh, A. Patel, P. and Gautam, U. S. (2011). Study of different plant growth retardants on flowering, fruiting, yield and economics of okra (Abelmoschus esculentus (L.) Moench). Progr. Hort., 43(1): 166-167.

Singh K. P. (2013). Effect of growth retardants on reproductive characters and yield of okra Cv. Parbhani Kranti. Indian J. Hort., 70(1): 154155.

Hossain M.D., Rahman M.A., Hoque M.M., Islam M.S. and Salam M.A. (2001). Year round okra production and economic return as influenced by spacing in Barisal Region. Bangladesh J. Agric. Res. 26, pp. 319-328.

Weimer, R. (1990). Agroecology. Mc Graw Hill Book Co. Inc., New York.

\section{How to cite this article:}

Jyothi, M. and Tambe, T. B. 2019. Effect of Plant Densities and Cycocel on Fruit and Yield Attributes of Okra (Abelmoschus Esculentus. (L.) Moench) Cv. Parbhani Kranti. Int.J.Curr.Microbiol.App.Sci. 8(10): 859-863. doi: https://doi.org/10.20546/ijcmas.2019.810.099 